\title{
Estudo de Riscos Cardiovasculares em Adolescentes (ERICA): fatores associados ao trabalho na adolescência
}

\author{
Study of Cardiovascular Risks in Adolescents (ERICA): \\ factors associated with work in adolescence
}

\begin{abstract}
Elisa Brosina de Leon (https://orcid.org/0000-0002-2559-6897) ${ }^{1}$
Bruno Mendes Tavares (https://orcid.org/0000-0003-0956-6484) ${ }^{2}$

Tiótrefis Gomes Fernandes (https://orcid.org/0000-0002-8563-9529) ${ }^{1}$

Roberta Lins Gonçalves (https://orcid.org/0000-0003-4976-0716) ${ }^{1}$

Fernanda Figueiroa Sanchez Franco (https://orcid.org/0000-0002-6362-4617) ${ }^{1}$

Marina Batista Chaves Azevedo de Souza (https://orcid.org/0000-0003-0704-0534) ${ }^{3}$

Frida Marina Fischer (https://orcid.org/0000-0001-9403-6300) ${ }^{4}$

Bárbara Iansã de Lima Barroso (https://orcid.org/0000-0002-3591-4350) ${ }^{5}$
\end{abstract}

${ }^{1}$ Faculdade de Educação Física e Fisioterapia, Universidade Federal do Amazonas (UFAM). Av. General Rodrigo Octávio 6200, Coroado I. 69080 900 Manaus AM Brasil. elisadleon@ufam.edu.br

${ }^{2}$ Instituto de Ciências Biológicas, UFAM. Manaus AM Brasil.

${ }^{3}$ Departamento de Terapia Ocupacional, Universidade Federal de Sergipe. Lagarto SE Brasil.

${ }^{4}$ Faculdade de Saúde

Pública, Universidade de São Paulo. São Paulo SP Brasil.

${ }^{5}$ Departamento de Terapia Ocupacional, Universidade Federal da Paraíba. João Pessoa PB Brasil.

\begin{abstract}
This study aimed to verify the association between work and sociodemographic factors, health behaviors, and cardiovascular risk in Brazilian adolescents. Data from the Study of Cardiovascular Risks in Adolescents (ERICA) were analyzed, with a sample of 37,815 adolescents aged 12-17 years and morning shift students. Considering complex sampling, crude and adjusted Prevalence Ratios (PR) were calculated with interval estimates for variables by Poisson multiple regression model with robust variance estimate. The sociodemographic factors associated with work among Brazilian adolescents were male gender (PR 1.34), aged 15-17 years, with maternal schooling up to elementary school (PR 1.26), public school students (PR 1.63), and from the rural area (PR 1.90). Regarding lifestyle habits and social behavior, the association was significant in physically active (PR 1.19) and alcohol-consuming students (PR 1.35). In conclusion, work among Brazilian adolescent morning shift students is still strongly linked to social issues. Work was associated with alcohol consumption, and there was no association with metabolic markers. Key words Child labor, Cardiovascular diseases, Adolescent working students
\end{abstract}

Resumo O objetivo do estudo foi verificar associação entre trabalho e fatores sociodemográficos, de condutas de saúde e de risco cardiovasculares em adolescentes brasileiros. Foram analisados dados do Estudo de Riscos Cardiovasculares em Adolescentes (ERICA), com amostra constituida de 37.815 adolescentes com idades entre 12 e 17 anos, estudantes do turno matutino. Considerando a amostragem complexa, foram calculadas as $R a$ zões de Prevalências (RP) brutas e ajustadas com estimativas intervalares para as variáveis usando modelo de regressão múltipla de Poisson com estimativa robusta da variância. Os fatores sociodemográficos associados ao trabalho entre adolescentes brasileiros foram: sexo masculino $(R P 1,34)$, idade entre 15 e 17 anos (RP 1,68), escolaridade materna até o fundamental (RP 1,26), de escola pública $(R P 1,63)$ e da zona rural $(R P 1,90)$. Em relação a hábitos de vida e comportamento social, a associação foi significativa em alunos ativos fisicamente $(R P 1,19)$ e que consumiam álcool $(R P$ 1,35). Em conclusão, o trabalho entre adolescentes brasileiros estudantes do turno matutino ainda está ligado fortemente às questões sociais. O trabalho foi associado ao consumo de álcool e não houve associação com marcadores metabólicos.

Palavras-chave Trabalho infantil, Doenças cardiovasculares, Trabalho em estudantes adolescentes 


\section{Introdução}

A Constituição Federal garante à população jovem ações que possam reduzir os riscos na infância e adolescência, como o trabalho extemporâneo. O artigo 224 prevê que "a política de atendimento dos direitos da criança e do adolescente far-se-á através de um conjunto articulado de ações governamentais e não governamentais, da União, dos Estados, do Distrito Federal e dos Municípios"'.

No entanto, estudos conduzidos com adolescentes e documentos importantes sobre $\mathrm{o}$ assunto identificam que ainda há um longo caminho a ser percorrido em termos de legislação e de políticas públicas, considerando que muitos adolescentes não têm outra alternativa a não ser o trabalho, principalmente os que residem em zonas rurais. É possível que a inserção e a permanência de adolescentes no trabalho estejam interligadas com as influências do meio familiar - a exemplo da formação educacional e sociocultural dos pais - e com as realidades socioeconômicas dos países e contextos em que vivem, algo que pode resultar em repercussões negativas para a saúde desses jovens $\mathrm{s}^{2-4}$.

Algumas questões que envolvem o trabalho na adolescência já foram mencionadas como negativas para a saúde dos sujeitos que as vivenciam como: alta exigência, pressão psicológica, privação de sono, diminuição de tempo para atividades de lazer e baixa qualidade da alimentação. Estudos indicam que características como essas, aumentam o risco de desenvolvimento de doenças crônicas não transmissíveis como, por exemplo, doenças cardiovasculares (DCV), obesidade, além de terem uma tendência ao uso de psicotrópicos ${ }^{5,6}$.

Nesse sentido, presume-se que os efeitos do trabalho precoce e as DCV podem ser sugestivos a: pressão arterial elevada, excesso de peso, dislipidemia, glicose elevada e síndrome metabólica, que são consideradas as principais causadoras de óbitos nos países ocidentais ${ }^{6}$ repercutindo em impactos socioeconômicos negativos para os próprios indivíduos, seus familiares e o Estado. Tais implicações podem gerar custos elevados com medicação, atendimentos médicos e de outros profissionais de saúde, internação, licença médica e aposentadorias precoces ${ }^{5,7-9}$.

Pesquisas científicas conduzidas nas últimas décadas em algumas cidades do Brasil, a exemplo de Belo Horizonte e Londrina, foram realizadas com o objetivo de analisar as condutas de saúde e a incidência de doenças cardiovasculares em adolescentes. Os resultados demonstraram o baixo consumo de frutas e verduras, além da inatividade física, como fatores de risco mais prevalentes, os quais se desdobram em doenças cardiovasculares $^{10-12}$. A associação entre as condutas de saúde e as DCV também vem sendo pesquisada internacionalmente, em diferentes contextos socioeconômicos, os quais identificaram a atividade de trabalho como potencial fator de risco para os indivíduos participantes das pesquisas ${ }^{11,13,14}$.

Embora alguns estudos brasileiros demonstrem associação entre os comportamentos de saúde e a incidência de doenças cardiovasculares nessa população, a literatura carece de pesquisas que investiguem a questão do trabalho na adolescência como um possível fator de risco. Além disso, acredita-se que pesquisas nacionais, atualizadas e com amostras representativas, poderiam contribuir de forma relevante na discussão sobre trabalho, juventude e saúde.

Este artigo é resultado da análise de dados do Estudo de Riscos Cardiovasculares em Adolescentes (ERICA), uma pesquisa pioneira no país, que já coletou dados de cerca de 75 mil jovens de 12 a 17 anos em todas as regiões do Brasil. O objetivo do ERICA é gerar evidências científicas que possam embasar e/ou reorientar políticas públicas de saúde direcionadas aos adolescentes e jovens brasileiros, já que seus resultados auxiliam na identificação das vulnerabilidades e necessidades de saúde dessa população.

Este estudo, especificamente, apresenta um recorte dos dados do ERICA e teve como objetivo verificar a associação entre trabalho e fatores sociodemográficos, condutas de saúde e riscos cardiovasculares em adolescentes brasileiros.

\section{Métodos}

A amostra foi composta por adolescentes participantes do Estudo de Riscos Cardiovasculares em Adolescentes (ERICA), uma pesquisa seccional, nacional, de base escolar. Os dados foram coletados entre fevereiro de 2013 e novembro de 2014, contemplando avaliações em todas as capitais brasileiras e cidades com mais de 100.000 habitantes. No geral, foram avaliados 85.615 estudantes de 12 a 17 anos de escolas públicas e privadas, localizadas em zonas urbanas e rurais do Brasil.

A população de pesquisa foi estratificada em 32 estratos geográficos, constituídos pelas $27 \mathrm{ca}$ pitais e por cinco conjuntos de municípios com mais de 100.000 habitantes de cada uma das cinco regiões do Brasil. O tamanho da amostra foi 
calculado para cada estrato, visando assegurar estimativas representativas para cada cidade, num total de 124 municípios.

As 1.247 escolas foram selecionadas com probabilidade proporcional, influenciada diretamente pelo número de alunos matriculados no ano precedente aos três últimos anos do ensino fundamental e nos três anos do ensino médio, e inversamente à distância em quilômetros entre a sede do município onde se localiza a escola e a sede do município da capital. As turmas de cada escola foram selecionadas em combinação de turnos (períodos da manhã e da tarde). Todos os alunos das turmas selecionadas foram convidados a participar do ERICA. Para a análise, foram excluídos adolescentes com deficiência física provisória ou permanente e grávidas. Mais detalhes sobre o processo de amostragem encontram-se na publicação de Vasconcellos et al. ${ }^{15}$.

Após o contato com a escola e aceite do diretor, foi aplicado o questionário individual aos adolescentes, estruturado e autopreenchido, inserido em um coletor eletrônico de dados Personal Digital Assistant (PDA), composto por onze blocos temáticos, aplicados em salas de aula, sob a supervisão da equipe do estudo previamente treinada. A logística e os métodos do ERICA foram descritos por Bloch et al. ${ }^{16}$.

Para determinar o nível de atividade física dos adolescentes, foi utilizada uma adaptação do Self-Administered Physical Activity Checklist ${ }^{17}$, formado por 24 modalidades que possibilitam a coleta de informações sobre frequência (dias) e o tempo (horas e minutos) em que praticou, na última semana, alguma das atividades listadas. A versão utilizada foi validada pelo ERICA $^{18}$. Os adolescentes que não acumularam, pelo menos, $300 \mathrm{~min} / \mathrm{semana}$ de atividade física foram considerados insuficientemente ativos inativos ${ }^{19} \mathrm{e}$ $\geq 300 \mathrm{~min} / \mathrm{sem}$ foram os ativos. A frequência de adolescentes que não referiram qualquer prática de atividade física na semana anterior à pesquisa (0 min/semana) também foi avaliada ${ }^{20}$.

Para a análise de comportamento tabágico e consumo de álcool foram considerados a intensidade e as características do consumo ${ }^{21}$. Considerou-se fumantes de cigarros de tabaco atuais ter consumido pelo menos uma vez nos últimos 30 dias. Para investigar o uso frequente do cigarro de tabaco, utilizou-se como indicador ter fumado cigarros por pelo menos sete dias seguidos ${ }^{22}$. Também foram utilizados os dados referentes ao consumo de bebidas alcoólicas. Essa variável foi aferida por meio das seguintes perguntas: (a) Idade em que tomou pelo menos um copo (ou dose) de bebida alcoólica pela primeira vez e (b) Dias de ingestão de pelo menos um copo (dose) de álcool, nos últimos 30 dias $^{23}$.

O bloco do sono foi composto de quatro perguntas que permitiram a determinação da quantidade de sono em dias de semana comum (dia útil) e no fim de semana ${ }^{24}$. As horas de sono em dias úteis foram estratificadas em três estratos: menos de seis horas; maior ou igual a seis horas e menos de oito horas; e maior ou igual a oito horas. O tempo de sono nos finais de semana foi estratificado em menos de oito horas e maior ou igual a oito horas.

Para as variáveis relativas ao trabalho, primeiramente foram consideradas as horas de trabalho atualmente por semana, categorizados em "não trabalha" (0 horas/semana), "trabalha até 20 horas/semana", "trabalha entre 21 e 40 horas/semana”, recebendo rendimentos ou não. Para analisar as associações, a variável foi dicotomizada entre "não trabalha" e "trabalha" (uma ou mais horas de trabalho por semana), esta última composta pela junção das duas últimas categorias da variável anterior.

Além do questionário, foram mensuradas as medidas antropométricas de estatura, peso, circunferência da cintura e do braço, seguida da avaliação da pressão arterial ${ }^{25}$. $\mathrm{O}$ estado nutricional foi determinado pelo índice de massa corporal (IMC) e classificado segundo referência da Organização Mundial de Saúde (OMS) ${ }^{26}$. A pressão arterial (PA) foi verificada com monitor digital (Omron 05-IT), validado para uso em adolescentes, e mensurada no braço direito. A criação dessa variável foi baseada nos critérios de classificação do Fourth Report on the Diagnosis, Evaluation, and Treatment of High Blood Pressure in Children and Adolescents (2004) ${ }^{27}$, considerando-se como hipertensão arterial os resultados de PA elevada e adolescentes não hipertensos os que apresentaram valores normais ou limítrofes, conforme descrito por Bloch et al. ${ }^{25}$.

A coleta de sangue exigia jejum de 12 horas, sendo, portanto, realizada somente em estudantes das turmas matutinas. Assim, a inferência dos resultados relativos à relação entre trabalho e as demais variáveis só pode ser feita para estudantes que estudam no turno da manhã. As amostras permitiram a dosagem de colesterol total, colesterol-hdl, triglicerídeos, glicose, hemoglobina glicada e insulina ${ }^{28}$. Os métodos de dosagem plasmáticos e pontos de corte e critérios para determinação de Síndrome Metabólica podem ser consultados nos artigos de Faria-Neto et al. ${ }^{29} \mathrm{e}$ Kuschnir et al. ${ }^{30}$. 


\section{Análise estatística}

A amostra do estudo foi composta de adolescentes que preencheram o bloco relativo ao trabalho e que possuíam dados de coleta de sangue. As prevalências de trabalho foram estimadas com os respectivos intervalos de 95\% de confiança (IC95\%), de acordo com os aspectos sociodemográficos e os hábitos de vida relativos à saúde.

O teste Qui-quadrado de Pearson foi utilizado para avaliar a diferença das prevalências da situação de trabalho de acordo com as demais variáveis, considerando o nível de significância de 0,05. Foram calculadas as Razões de Prevalência (RP) brutas com estimativas de IC95\% para cada variável, usando o modelo de Poisson com variância robusta, assim como para o modelo de regressão múltipla, controlando por possíveis variáveis de confundimento. Para a análise multivariada foram incluídas as variáveis que apresentaram valor de " $p$ " menor do que 0,20 na análise univariada, cujos critérios para entrada de variáveis seguiram o modelo hierarquizado a partir de três grupos: (1) sociodemográfico, (2) hábitos de vida e (3) relativo à saúde. As variáveis foram ajustadas por aquelas pertencentes ao mesmo grupo e por aquelas que se apresentaram significativas $(\mathrm{p}<0,05)$ no(s) grupo(s) anteriore(s), e o modelo final foi composto por aquelas que apresentaram significância estatística após o último grupo de análise. Os dados foram analisados no programa Stata, versão 14, utilizando-se o módulo Survey (com fatores de ponderação) para analisar dados de amostra complexa.

O projeto ERICA foi aprovado pelo Comitê de Ética em Pesquisa de cada uma das 27 unidades da federação. Todos os adolescentes assinaram o termo de assentimento e, em cinco estados, foi adicionado o termo de consentimento livre e esclarecido assinado pelos responsáveis. $\mathrm{O}$ projeto recebeu o aval das Secretarias Estaduais e Municipais de Educação e Saúde.

\section{Resultados}

Dos 85.615 estudantes que participaram do ERICA em todo o Brasil, foram analisados os dados de 37.815 adolescentes que apresentavam informações sobre as variáveis "trabalho", "estado nutricional”, "pressão arterial” e "exames bioquímicos". Todos os adolescentes estudavam no turno matutino, dos quais $60,0 \%$ eram do sexo feminino e 54,2\% tinham entre 15 e17 anos, com média de 14,6 (DP=1,6) anos de idade. A maioria (74\%) estudava em escolas públicas e era das Regiões Nordeste (31\%) e Sudeste (22,7\%), seguidos das Regiões Norte (19,3\%), Centro-Oeste (14,5\%) e Sul (12,5\%).

A prevalência de adolescentes que declararam trabalhar no último ano foi de $16,2 \%$, sendo poucos (1\%) acima de 20 horas semanais. Em relação a aspectos sociodemográficos, meninos brasileiros trabalhavam mais, nas duas categorias de horas, comparados com as meninas (RP1: 1,45 e RP2: 1,55, respectivamente). Adolescentes mais velhos reportaram ter trabalhado mais comparados com os mais novos, principalmente os que trabalhavam entre 21 e 40 horas semanais (RP2: 11,9) (Tabela 1).

Adolescentes filhos de mães com menos escolaridade trabalhavam mais, considerando menor carga de trabalho (RP1: 1,67), porém eram os que menos trabalhavam de 21 a 40h/semana (RP2: 0,46). A frequência de adolescentes trabalhadores foi maior nas escolas públicas do que nas privadas em ambas as cargas horárias de trabalho semanal (RP1: 1,98 e RP2: 3,27, respectivamente) e maior na zona rural até 20 horas (RP1: 2,20), e menos, entre 21 e 40 horas (RP2: 0,15). As regiões que concentraram um maior número de estudantes trabalhadores foram a Centro-Oeste, a Sudeste e a Sul, em ambas as cargas horárias (Tabela 1 ).

Com relação aos aspectos relacionados a hábitos de vida, os adolescentes que mais trabalhavam referiram dormir menos de oito horas diárias tanto em dias úteis, quanto nos finais de semana. Entre os que trabalhavam até 20 horas, observou-se uma frequência maior de adolescentes considerados ativos fisicamente (RP1 1,48); o mesmo não foi observado entre quem referiu trabalhar entre 21 e 40 horas. Os adolescentes que trabalhavam menos disseram que fumavam e bebiam mais (RP1: 1,44 e RP1:1,62, respectivamente). Entre os que mais trabalhavam, somente o hábito de consumir álcool foi significativo (RP2: 2,05) (Tabela 1).

Por fim, considerando as informações relativas à saúde, adolescentes abaixo do peso trabalhavam menos em ambas as cargas horárias de trabalho (RP1: 0,54 e RP2: 0,13), e os estudantes com sobrepeso trabalhavam até 20 horas semanais (RP1: 0,83), quando comparados com os de peso adequado. A prevalência de estudantes com hipertensão arterial, síndrome metabólica e colesterol total elevado foi menor nos que trabalhavam de 21 a 40h/semana (Tabela 1). 
Tabela 1. Prevalências e Razões de Prevalência (RP) de trabalho no último ano em adolescentes brasileiros de 12 a 17 anos, considerando amostragem complexa, por variáveis sociodemográficas, hábitos de vida e relativos à saúde. Brasil, 2013-2014 (n=37.815).

\begin{tabular}{|c|c|c|c|c|c|c|}
\hline \multirow[b]{2}{*}{ Variáveis } & \multicolumn{5}{|c|}{ Prevalências \% (IC95\%) } & \multirow[b]{2}{*}{$\mathbf{P}^{\star}$} \\
\hline & Não trabalha & $\begin{array}{c}\text { Trabalha até } 20 \\
\text { horas/semana }(1)\end{array}$ & RP $1^{\dagger}(\mathrm{IC} 95 \%)$ & $\begin{array}{l}\text { Trabalha } \\
\text { entre 21- } \\
40 \text { horas/ } \\
\text { semana }(2)\end{array}$ & RP $2^{\dagger \dagger}($ IC95\%) & \\
\hline Total & $82,5(80,9-84,1)$ & $15,2(13,8-16,8)$ & & $1,0(0,6-1,4)$ & & \\
\hline \multicolumn{7}{|l|}{ Sociodemográficas } \\
\hline Sexo & & & & & & $<0,001$ \\
\hline Feminino & $85,7(84,6-86,7)$ & $12,5(11,5-13,6)$ & 1 & $1,8(1,3-2,4)$ & 1 & \\
\hline Masculino & $79,4(76,6-81,9)$ & $18,0(15,5-20,8)$ & $1,45(1,25-1,68)$ & $2,6(2,0-3,5)$ & $1,55(1,18-2,03)$ & \\
\hline Idade & & & & & & $<0,001$ \\
\hline 12 a 14 anos & $87,7(85,9-89,4)$ & $11,9(10,2-13,8)$ & 1 & $0,3(0,2-0,5)$ & 1 & \\
\hline 15 a 17 anos & $78,0(76,1-79,8)$ & $18,2(16,5-19,9)$ & $1,59(1,40-1,81)$ & $3,8(2,9-5,0)$ & $11,9(6,90-20,50)$ & \\
\hline Escolaridade da Mãe & & & & & & $<0,001$ \\
\hline $\begin{array}{l}\text { Até fundamental } \\
\text { incompleto }\end{array}$ & $76,4(71,0-81,1)$ & $22,5(17,8-28,1)$ & $1,67(1,33-2,11)$ & $1,1(0,5-2,1)$ & $0,46(0,23-0,91)$ & \\
\hline $\begin{array}{l}\text { Fund. Completo a médio } \\
\text { incompleto }\end{array}$ & $78,7(75,6-81,4)$ & $18,3(15,4-21,7)$ & $1,38(1,17-1,62)$ & $3,0(2,3-3,9)$ & $1,29(0,86-1,95)$ & \\
\hline $\begin{array}{l}\text { Médio completo a Superior } \\
\text { completo }\end{array}$ & $84,3(82,6-85,8)$ & $13,3(12,0-14,7)$ & 1 & $2,4(1,7-3,5)$ & 1 & \\
\hline Tipo de escola & & & & & & $<0,001$ \\
\hline Pública & $80,2(78,4-82,0)$ & $17,2(15,4-19,0)$ & $1,98(1,60-2,44)$ & $2,6(1,9-3,5)$ & $3,27(1,61-6,65)$ & \\
\hline Privada & $90,4(88,4-92,1)$ & $8,8(7,4-10,4)$ & 1 & $0,9(0,5-1,5)$ & 1 & \\
\hline $\begin{array}{l}\text { Ambiente geográfico da } \\
\text { escola }\end{array}$ & & & & & & $<0,001$ \\
\hline Urbano & $83,2(82,0-84,4)$ & $14,5(13,4-15,6)$ & 1 & $2,3(1,8-3,0)$ & 1 & \\
\hline Rural & $66,9(60,5-72,8)$ & $32,8(26,7-39,6)$ & $2,20(1,76-2,74)$ & $0,3(0,5-1,3)$ & $0,15-(0,03-0,75)$ & \\
\hline Região do Brasil & & & & & & $<0,001$ \\
\hline Nordeste & $88,8(87,4-90,0)$ & $10,2(9,0-11,4)$ & 1 & $1,1(0,6-2,0)$ & 1 & \\
\hline Norte & $87,0(85,9-88,1)$ & $12,0(11,1-13,0)$ & $1.18(1,03-1,36)$ & $0,9(0,6-1,4)$ & $0,89(0,43-1,81)$ & \\
\hline Sudeste & $80,9(77,9-83,5)$ & $16,7(14,1-19,6)$ & $1,67(1,37-2,04)$ & $2,4(1,6-3,8)$ & $2,52(1,21-5,25)$ & \\
\hline Sul & $80,2(77,0-83,0)$ & $16,2(13,9-18,7)$ & $1,65(1,36-2,00)$ & $3,7(2,7-5,0)$ & $3,68(1,84-7,38)$ & \\
\hline Centro-Oeste & $77,7(74,4-80,7)$ & $20,1(16,9-23,9)$ & $2,01(1,64-2,47)$ & $2,1(1,2-3,5)$ & $2,36(1,12-4,97)$ & \\
\hline \multicolumn{7}{|l|}{ Hábitos de vida } \\
\hline Sono dias úteis & & & & & & $<0,001$ \\
\hline$<6$ horas & $79,0(76,3-81,5)$ & $16,4(14,0-19,2)$ & $1,04(0,83-1,32)$ & $4,6(3,1-6,8)$ & $3,76(2,40-5,89)$ & \\
\hline$\geq 6$ horas e $<8$ horas & $83,5(81,8-85,0)$ & $13,8(12,4-15,3)$ & $0,86(0,70-1,06)$ & $2,7(1,9-3,9)$ & $2,20(1,40-3,47)$ & \\
\hline$\geq 8$ horas & $82,3(80,9-84,1)$ & $16,5(13,7-19,6)$ & 1 & $1,2(0,9-1,6)$ & 1 & \\
\hline Sono fim de semana & & & & & & 0,003 \\
\hline$<8$ horas & $78,9(75,9-81,6)$ & $17,3(14,6-20,5)$ & $1,19(1,00-1,40)$ & $3,8(1,6-5,4)$ & $1,95(1,25-3,05)$ & \\
\hline$\geq 8$ horas & $83,1(81,4-84,6)$ & $15,0(13,4-16,6)$ & 1 & $2,0(1,5-2,7)$ & 1 & \\
\hline Atividade Física & & & & & & $<0,001$ \\
\hline Inativo $(0 \mathrm{~min} / \mathrm{sem})$ & $85,0(83,4-86,5)$ & $12,4(11,1-13,9)$ & 1 & $2,6(1,8-3,7)$ & 1 & \\
\hline $\begin{array}{l}\text { Insuficiente ativo ( } 0 \text { a } 300 \\
\mathrm{~min} / \mathrm{sem})\end{array}$ & $85,0(81,5-88,0)$ & $12,8(10,0-16,2)$ & $1,02(0,77-1,35)$ & $2,2(1,5-3,4)$ & $0,86(0,65-1,15)$ & \\
\hline Ativo $(\geq 300 \mathrm{~min} / \mathrm{sem})$ & $79,6(77,6-81,4)$ & $18,5(16,7-20,4)$ & $1,48(1,26-1,73)$ & $2,0(1,5-2,5)$ & $0,78(0,55-1,11)$ & \\
\hline Tabagismo & & & & & & 0,04 \\
\hline Fuma atualmente & $73,9(66,2-80,3)$ & $21,4(16,0-28,0)$ & $1,44(1,07-1,93)$ & $4,7(1,6-13,0)$ & $2,44(0,85-7,05)$ & \\
\hline Não fuma atualmente & $82,7(81,0-84,2)$ & $15,1(13,6-16,8)$ & 1 & $2,2(1,7-2,8)$ & 1 & \\
\hline
\end{tabular}


Tabela 1. Prevalências e Razões de Prevalência (RP) de trabalho no último ano em adolescentes brasileiros de 12 a 17 anos, considerando amostragem complexa, por variáveis sociodemográficas, hábitos de vida e relativos à saúde. Brasil, 2013-2014 (n=37.815).

\begin{tabular}{|c|c|c|c|c|c|c|}
\hline \multirow[b]{2}{*}{ Variáveis } & \multicolumn{5}{|c|}{ Prevalências \% (IC95\%) } & \multirow[b]{2}{*}{$\mathbf{P}^{*}$} \\
\hline & Não trabalha & $\begin{array}{c}\text { Trabalha até } 20 \\
\text { horas/semana (1) }\end{array}$ & RP $1^{\dagger}(\mathrm{IC} 95 \%)$ & $\begin{array}{l}\text { Trabalha } \\
\text { entre } 21- \\
40 \text { horas/ } \\
\text { semana }(2)\end{array}$ & RP $2^{\dagger \dagger}($ IC95\%) & \\
\hline Álcool & & & & & & $<0,001$ \\
\hline Bebe & $75,1(71,3-78,6)$ & $21,3(17,9-25,2)$ & $1,62(1,38-1,90)$ & $3,6(2,6-4,8)$ & $2,05(1,51-2,77)$ & \\
\hline Não bebe & $84,8(83,7-85,8)$ & $13,4(12,3-14,5)$ & 1 & $1,9(1,4-2,5)$ & 1 & \\
\hline \multicolumn{7}{|l|}{ Relativo à Saúde } \\
\hline Transtorno Mental Comum & & & & & & 0,15 \\
\hline Não & $83,1(81,2-84,9)$ & $14,7(12,9-16,6)$ & 1 & $2,2(1,6-3,0)$ & 1 & \\
\hline Sim & $81,2(79,3-83,0)$ & $16,6(14,8-18,5)$ & $1,12(0,97-1,30)$ & $2,2(1,7-2,8)$ & $0,99(0,74-1,33)$ & \\
\hline Estado Nutricional & & & & & & 0,003 \\
\hline Abaixo do peso & $91,0(85,9-94,4)$ & $8,7(5,3-13,9)$ & $0,54(0,33-0,87)$ & $0,3(0,2-0,7)$ & $0,13(0,06-0,29)$ & \\
\hline Adequado & $81,7(80,0-83,2)$ & $15,9(14,4-17,6)$ & 1 & $2,4(1,8-3,1)$ & 1 & \\
\hline Sobrepeso & $84,6(82,0-86,8)$ & $13,4(11,3-15,7)$ & $0,83(0,73-0,96)$ & $2,1(1,3-3,2)$ & $0,84(0,59-1,19)$ & \\
\hline Obeso & $83,2(79,9-86,0)$ & $15,1(12,4-18,4)$ & $0,94(0,77-1,16)$ & $1,7(1,1-2,6)$ & $0,69(0,42-1,14)$ & \\
\hline Hipertensão arterial & & & & & & 0,06 \\
\hline Sim & $82,5(81,0-84,0)$ & $16,1(13,2-19,4)$ & $1,05(0,90-1,22)$ & $1,3(0,8-2,1)$ & $0,52(0,31-0,87)$ & \\
\hline Não & $82,7(79,4-85,5)$ & $15,2(13,7-16,7)$ & 1 & $2,3(1,8-3,0)$ & 1 & \\
\hline Glicemia elevada & & & & & & 0,06 \\
\hline Sim & $82,3(80,7-83,9)$ & $10,4(7,8-13,8)$ & $0,67(0,49-0,92)$ & $2,6(1,2-5,6)$ & $1,12(0,51-2,45)$ & \\
\hline Não & $87,0(82,7-90,4)$ & $15,5(13,9-17,2)$ & 1 & $2,2(1,7-2,9)$ & 1 & \\
\hline Síndrome Metabólica & & & & & & 0,23 \\
\hline Sim & $82,5(76,6-87,1)$ & $15,2(12,1-22,6)$ & $1,07(0,76-1,53)$ & $0,8(0,4-1,6)$ & $0,33(0,15-0,75)$ & \\
\hline Não & $82,5(80,8-84,0)$ & $15,2(13,7-16,9)$ & 1 & $2,3(1,7-3,0)$ & 1 & \\
\hline Colesterol total & & & & & & 0,21 \\
\hline Adequado & $81,7(80,1-83,2)$ & $15,7(14,3-17,1)$ & 1 & $2,6(1,9-3,6)$ & 1 & \\
\hline Limítrofe & $83,3(79,7-86,5)$ & $14,9(11,8-18,7)$ & $0,95(0,75-1,18)$ & $1,8(1,3-2,4)$ & $0,69(0,44-1,08)$ & \\
\hline Elevado & $83,8(81,7-85,8)$ & $14,5(12,5-16,7)$ & $0,91(0,79-1,05)$ & $1,7(1,1-2,5)$ & $0,62(0,43-0,89)$ & \\
\hline HDL & & & & & & 0,71 \\
\hline Adequado & $82,4(80,0-84,5)$ & $15,5(13,4-17,9)$ & 1 & $2,1(1,5-2,9)$ & 1 & \\
\hline Inadequado & $82,8(81,2-84,2)$ & $14,9(13,4-16,5)$ & $0,96(0,83-1,12)$ & $2,3(1,8-3,1)$ & $1,11(0,82-1,50)$ & \\
\hline LDL & & & & & & 0,46 \\
\hline Adequado & $82,1(80,5-83,7)$ & $15,5(14,1-17,1)$ & 1 & $2,4(1,7-3,2)$ & 1 & \\
\hline Limítrofe & $83,6(80,4-86,3)$ & $14,6(11,9-17,9)$ & $0,94(0,78-1,12)$ & $1,8(1,3-2,6)$ & $0,76(0,49-1,18)$ & \\
\hline Elevado & $85,3(80,8-88,8)$ & $13,0(9,9-17,0)$ & $0,84(0,63-1,11)$ & $1,7(0,8-3,8)$ & $0,68(0,31-1,48)$ & \\
\hline Triglicerídeos & & & & & & 0,26 \\
\hline Adequado & $82,0(80,0-83,9)$ & $15,6(13,7-17,7)$ & 1 & $2,4(1,8-3,1)$ & 1 & \\
\hline Limítrofe & $84,9(82,2-87,2)$ & $13,6(11,3-16,3)$ & $0,86(0,67-1,10)$ & $1,5(0,9-2,6)$ & $0,62(0,40-0,97)$ & \\
\hline Elevado & $84,3(81,4-86,2)$ & $13,8(11,4-16,6)$ & $0,87(0,70-1,09)$ & $1,9(1,0-3,5)$ & $0,76(0,39-1,47)$ & \\
\hline Circunferência da cintura & & & & & & 0,61 \\
\hline Adequada & $82,5(80,8-84,1)$ & $15,2(13,6-16,9)$ & 1 & $2,3(1,7-3,0)$ & 1 & \\
\hline Elevada & $82,7(80-85,0)$ & $15,5(13,2-18,2)$ & $1,01(0,86-1,20)$ & $1,8(1,3-2,6)$ & $0,77(0,51-1,17)$ & \\
\hline
\end{tabular}


Após a análise multivariada para amostras complexas, os fatores sociodemográficos associados ao trabalho entre adolescentes brasileiros em qualquer carga horária foram: sexo masculino (RP: 1,34; IC95\%: 1,17-1,53); alunos mais velhos (RP: 1,68; IC95\%: 1,45-1,94); escolaridade materna até o fundamental incompleto (RP: 1,26; IC95\%: 1,08-1,47) em comparação com as que tinham, pelo menos, o ensino médio; estudantes de escola pública (RP: 1,63; IC95\%: 1,34-1,99); localizados na zona rural (RP: 1,90; IC95\%: 1,522,38); residentes em região diferente da Nordeste, com destaque para a Região Centro-Oeste (RP: 1,81; IC95\%: 1,55-2,12, Tabela 2).

Em relação a hábitos de vida e comportamento social, a associação foi significativa em alunos ativos fisicamente (RP: 1,19; IC95\%: 1,06$1,33)$, em comparação com os inativos e que consumiam álcool regularmente (RP: 1,35; IC95\%: 1,20-1,52). O trabalho teve uma associação inversa em relação ao baixo peso (RP: 0,55; IC95\%: 0,35-0,89) (Tabela 2).

\section{Discussão}

O ERICA é um importante estudo epidemiológico sobre a saúde do adolescente brasileiro. Para este recorte, verificou-se que o trabalho está fortemente associado ao sexo masculino e em adolescentes mais velhos. $\mathrm{O}$ trabalho mostrou-se mais prevalente em estudantes de escolas públicas, moradores de área rural e com mães com menor escolaridade. Além disso, mostrou interferir na quantidade de sono diária e esteve associado a comportamentos de risco na adolescência e ao consumo de álcool, entretanto menos presente entre os sedentários ou com baixo peso ${ }^{9,10}$.

Crianças e adolescentes residentes em países em desenvolvimento comumente se encontram em situação econômica desfavorável e consequente vulnerabilidade social, o que acarreta maior necessidade de adentrar ao mercado de trabalho com o objetivo principal de contribuir com a renda familiar ou o orçamento domésti$\mathrm{co}^{31,32}$. Muitas vezes, a cobrança familiar sobre o ingresso no mercado de trabalho recai sobre os

Tabela 2. Modelo de regressão múltipla de Poisson: variáveis associadas ao trabalho em adolescentes brasileiros de 12 a 17 anos, considerando amostragem complexa. Brasil, 2013-2014 ( $\mathrm{n}=37.815)$.

\begin{tabular}{|c|c|c|c|c|}
\hline Variáveis & RP bruta & IC95\% & RP ajustada* & IC95\% \\
\hline \multicolumn{5}{|l|}{ Grupo 1 - Sociodemográficas } \\
\hline \multicolumn{5}{|l|}{ Sexo } \\
\hline Feminino & 1 & & 1 & \\
\hline Masculino & 1,43 & $(1,25-1,63)$ & $1,34^{\mathrm{a}}$ & $(1,17-1,53)$ \\
\hline \multicolumn{5}{|l|}{ Idade } \\
\hline 12 a 14 anos & 1 & & 1 & \\
\hline 15 a 17 anos & 1,60 & $(1,41-1,81)$ & $1,68^{\mathrm{a}}$ & $(1,45-1,94)$ \\
\hline \multicolumn{5}{|l|}{ Escolaridade da Mãe } \\
\hline Até fundamental incompleto & 1,47 & $(1,21-1,79)$ & $1,26^{\mathrm{a}}$ & $(1,08-1,47)$ \\
\hline Fund. Completo a médio incompleto & 1,36 & $(1,17-1,58)$ & $1,08^{\mathrm{a}}$ & $(0,98-1,20)$ \\
\hline Médio completo a Superior completo & 1 & & 1 & \\
\hline \multicolumn{5}{|l|}{ Tipo de escola } \\
\hline Pública & 2,09 & $(1,71-2,56)$ & $1,63^{\mathrm{a}}$ & $(1,34-1,99)$ \\
\hline Privada & 1 & & 1 & \\
\hline \multicolumn{5}{|l|}{ Ambiente geográfico da escola } \\
\hline Urbano & 1 & & 1 & \\
\hline Rural & 2,07 & $(1,70-2,52)$ & $1,9^{\mathrm{a}}$ & $(1,52-2,38)$ \\
\hline \multicolumn{5}{|l|}{ Região do Brasil } \\
\hline Nordeste & 1 & & 1 & \\
\hline Norte & 1,14 & $(0,94-1,39)$ & $1,20^{\mathrm{a}}$ & $(1,04-1,38)$ \\
\hline Centro-Oeste & 1,75 & $(1,41-2,16)$ & $1,81^{\mathrm{a}}$ & $(1,55-2,12)$ \\
\hline Sudeste & 1,56 & $(1,23-1,98)$ & $1,57^{\mathrm{a}}$ & $(1,34-1,84)$ \\
\hline Sul & 1,57 & $(1,25-1,97)$ & $1,57^{\mathrm{a}}$ & $(1,29-1,90)$ \\
\hline
\end{tabular}

continua 
Tabela 2. Modelo de regressão múltipla de Poisson: variáveis associadas ao trabalho em adolescentes brasileiros de 12 a 17 anos, considerando amostragem complexa. Brasil, 2013-2014 ( $\mathrm{n}=37.815)$.

\begin{tabular}{|c|c|c|c|c|}
\hline Variáveis & RP bruta & IC95\% & RP ajustada ${ }^{*}$ & IC95\% \\
\hline \multicolumn{5}{|l|}{ Grupo 2 - Hábitos de vida } \\
\hline \multicolumn{5}{|l|}{ Atividade Física } \\
\hline Inativo (0 $\mathrm{min} / \mathrm{sem})$ & 1 & & 1 & \\
\hline Insuficientemente ativo (0 a $300 \mathrm{~min} / \mathrm{sem})$ & 1,02 & $(0,79-1,31)$ & $1,03^{\mathrm{a}}$ & $(0,86-1,24)$ \\
\hline Ativo ( $\geq 300 \mathrm{~min} / \mathrm{sem})$ & 1,35 & $(1,18-1,54)$ & $1,19^{\mathrm{a}}$ & $(1,06-1,33)$ \\
\hline \multicolumn{5}{|l|}{ Álcool } \\
\hline Bebe & 1,57 & $(1,35-1,82)$ & $1,35^{\mathrm{a}}$ & $(1,20-1,52)$ \\
\hline Não bebe & 1 & & 1 & \\
\hline \multicolumn{5}{|l|}{ Tabagismo } \\
\hline Fuma atualmente & 1,61 & $(1,27-2,05)$ & 1,14 & $(0,87-1,49)$ \\
\hline Não fuma atualmente & 1 & & 1 & \\
\hline \multicolumn{5}{|l|}{ Sono em fim de semana } \\
\hline$<8$ horas & 1,19 & $(1,05-1,33)$ & 1,09 & $(0,96-1,24)$ \\
\hline$\geq 8$ horas & 1 & & 1 & \\
\hline \multicolumn{5}{|l|}{ Grupo 3 - Relativo à Saúde } \\
\hline \multicolumn{5}{|l|}{ Estado Nutricional } \\
\hline Abaixo do peso & 0,51 & $(0,34-0,76)$ & $0,56^{\mathrm{a}}$ & $(0,35-0,89)$ \\
\hline Adequado & 1 & & 1 & \\
\hline Sobrepeso & 0,83 & $(0,75-0,93)$ & $0,99^{\mathrm{a}}$ & $(0,86-1,13)$ \\
\hline Obeso & 0,91 & $(0,76-1,78)$ & $1,16^{\mathrm{a}}$ & $(0,97-1,38)$ \\
\hline \multicolumn{5}{|l|}{ Transtorno Mental Comum } \\
\hline Não & 1 & & 1 & \\
\hline Sim & 1,11 & $(0,98-1,26)$ & 1,11 & $(0,97-1,27)$ \\
\hline \multicolumn{5}{|l|}{ LDL } \\
\hline Adequado & 1,14 & $(1,01-1,71)$ & 1,13 & $(0,85-1,50)$ \\
\hline Limítrofe & 1,01 & $(0,88-1,58)$ & 0,99 & $(0,74-1,32)$ \\
\hline Elevado & 1 & & 1 & \\
\hline \multicolumn{5}{|l|}{ Triglicerídeos } \\
\hline Adequado & 1,14 & $(0,94-1,39)$ & 1,04 & $(0,86-1,27)$ \\
\hline Limítrofe & 1,01 & $(0,84-1,21)$ & 1,00 & $(0,77-1,30)$ \\
\hline Elevado & 1 & & 1 & \\
\hline
\end{tabular}

${ }^{\star}$ Ajuste por todas as variáveis do mesmo grupo e com aquelas variáveis significativas $(\mathrm{p}<0,05)$ do $(\mathrm{s})$ grupo(s) anteriores. ${ }^{\mathrm{a}}$ Valores apresentados pelas variáveis no modelo final (apenas com as variáveis estatisticamente significativas).

Fonte: Dados da Pesquisa, 2013 e 2014.

adolescentes do sexo masculino, com frequência na faixa etária que antecede o início da maiorida$\mathrm{de}^{33}$. Essa afirmação corrobora os dados apresentados neste estudo. Fatores culturais influenciam a forma como o trabalho infanto-juvenil é visto e, até mesmo, desejável. Evidências observadas na tendência mundial apontam que os meninos representam $52 \%$ de todas as crianças trabalhadoras na faixa etária de 12 a 14 anos, e na faixa etária de 15 a 17 anos, o aumento chega até $81 \% \%^{8,11-14,34}$.

Em uma parcela representativa da amostra, foi constatada baixa escolaridade materna $(\mathrm{RP}=1,26$; 1,08-1,47). Apesar de muitos lares brasileiros terem os pais como responsáveis pelo equilíbrio financeiro da residência, em grande parte deles, as mães são encarregadas de manter o núcleo familiar, como provedoras e incentivadoras dos filhos na continuidade dos estudos ${ }^{35}$. Muitos adolescentes conciliam o estudo com o trabalho precoce, porém a dedicação exclusiva a atividades escolares demonstra-se favorável a resultados que se traduzem em um futuro profissional possivelmente mais próspero ${ }^{4,36}$. Na maioria das vezes, a entrada mais cedo no mercado de trabalho está vinculada à desistência dos estudos, o que os leva, sistematicamente, a contribuírem com o aumento no setor de serviços do trabalho assalariado, subcontratado, terceirizado e informal ${ }^{10}$. 
Em relação aos dados demográficos, ressalta-se que a Região Nordeste, na última década, apresentava a maior proporção de jovens trabalhadores ou que estavam em busca de emprego. Essa região ainda continua sendo a de menor distribuição de renda, menor Produto Interno Bruto (PIB), menor Índice de Desenvolvimento Humano (IDH) e, consequentemente, maior concentração de pobreza ${ }^{37}$. No entanto, nossos dados demonstraram uma maior prevalência de trabalhadores adolescentes na Região Centro-Oeste, onde, tanto a agricultura de subsistência, complementar à pecuária e o extrativismo, quanto a agricultura comercial vêm crescendo nas últimas duas décadas ${ }^{38}$, apoiada, inclusive, por um quantitativo de mão de obra adolescente e morador da zona rural.

No grupo dos adolescentes que trabalhavam menos do que 20 horas semanais, houve um efeito protetor em relação ao peso corporal, similar ao encontrado no estudo de Drake et al. ${ }^{39}$. Ao se conceituar atividade física como qualquer movimento corporal produzido por músculos esqueléticos que resulte em gasto energético ${ }^{40}$, pode-se sugerir que o trabalho contribui para o gasto calórico, mantém os trabalhadores fisicamente ativos e é favorável à manutenção do peso dessas pessoas, no entanto isso não deve ser considerado como uma forma de incentivo ao trabalho precoce de crianças e adolescentes ${ }^{4}$.

Em relação ao consumo de bebidas alcoólicas, comportamento de risco que frequentemente tem início na infância ou na adolescência, é um grave problema de saúde pública. Apesar da proibição comercial garantida por lei, no Brasil, constata-se grande acessibilidade dos jovens aos psicotrópicos ${ }^{33,41,42}$. Este estudo observou associação positiva entre o fato de os adolescentes trabalharem e consumirem bebida alcoólica $(\mathrm{RP}=1,36$; 1,04-1,31). Uma situação análoga foi encontrada em estudo de Souza et al. ${ }^{33}$ no qual o consumo de álcool entre os estudantes trabalhadores foi de $81,0 \%$ e alcoolismo em $13,4 \%$ da amostra. Os dados são considerados alarmantes, pois se sabe que quanto mais cedo a ingestão de álcool e de outros psicotrópicos, maiores são as chances de abuso e dependência ${ }^{33,42}$.

Outro comportamento de risco é a restrição do número de horas diárias de sono, conforme demonstrado em nosso estudo. Os resultados deste estudo corroboram os dados de Felden et al. ${ }^{43}$ no qual demonstraram que os trabalhadores adolescentes apresentaram maior probabilidade de ter sono curto em comparação aos que não trabalharam. Os adolescentes que estudam no turno matutino necessitam acordar cedo e esse fator repercute em privação crônica do sono durante a semana e fadiga cumulativa, causando alterações no ciclo sono-vigília ${ }^{9,36,43,44}$. Esse fato pode conduzir a muitas disfunções orgânicas, como doença cardiovascular, déficits imunológicos, distúrbios no comportamento cognitivo e afetivo e risco de obesidade. Pode, ainda, ser precursor de hábitos deletérios, como o de consumir bebida alcoólica e tabaco ${ }^{45-48}$. Devido a todos os possíveis desdobramentos advindos da jornada de trabalho precoce, deve-se atentar para as repercussões na vida adulta provenientes das alterações no ritmo circadiano de sono e vigília.

A literatura aponta a obesidade como um importante fator de risco para o aparecimento de DCV, como hipertensão arterial em adolescentes ${ }^{49,50}$, acrescida de hábitos alimentares aterogênicos, comportamento sedentário e inatividade ${ }^{51}$. Na relação entre as variáveis de saúde analisadas (hipertensão arterial, síndrome metabólica e alterações bioquímicas) e as atividades de trabalho, não foram encontrados resultados estatísticos significativos. Esses achados podem estar relacionados à manutenção do peso corporal induzida pela prática de atividade física relatada pelos participantes.

Uma das limitações deste estudo se deve ao fato de só terem participado da coleta de sangue os jovens que estudavam no período da manhã, justificada pela necessidade de realização de jejum. Outra limitação pode ter ocorrido no uso de questionário para mensurar os hábitos de vida em adolescentes. No entanto, estratégia semelhante foi utilizada por outro grande estudo nacional, o estudo PeNSE ${ }^{52}$. Apesar de o estudo transversal resguardar limites quanto à incapacidade de estabelecer relação de causa e efeito, mostrou-se robusto para levantar hipóteses sobre o fenômeno do trabalho em adolescentes brasileiros. O tamanho amostral e o processo de amostragem complexa conferiram boa capacidade de validade externa dos achados.

Assim, o estudo evidenciou que o trabalho entre adolescentes brasileiros, em especial os que estudam no turno matutino, ainda está ligado fortemente a questões sociais, como alunos de baixa escolaridade materna, oriundos de escola pública e que moram na área rural. Apesar do trabalho ter estado associado ao consumo de álcool na adolescência, houve relação entre a proteção para baixo peso e o sedentarismo. $O$ trabalho na adolescência não esteve associado a marcadores cardiometabólicos. 


\section{Colaboradores}

EB Leon: concepção do projeto, coleta de dados no estado do Amazonas, redação do artigo e aprovação final da versão a ser publicada. BM Tavares, TG Fernandes, FM Fischer e BIL Barroso: concepção do projeto, redação do artigo e aprovação final da versão a ser publicada. RL Gonçalves, FFS Franco e MBCA Souza: concepção e redação do artigo e aprovação final da versão a ser publicada.

\section{Financiamento}

Ministério da Saúde (Departamento de Ciências e Tecnologia), Ministério da Ciência, Tecnologia e Inovação (Financiadora de Estudos e Projetos - FINEP e Conselho Nacional de Pesquisa CNPq).

\section{Referências}

1. Brasil. Lei $n^{\circ} 8.069$, de 13 de julho de 1990. Dispõe sobre o Estatuto da Criança e do Adolescente e dá outras providências. Diário Oficial da União 1990; 16 jul.

2. Guerreiro MD, Abrantes P. Como tornar-se adulto: processos de transição na modernidade avançada. Rev Bras Cien Soc 2005; 20(58):157-175.

3. Oliveira BRG, Robazzi MLCC. O trabalho na vida dos adolescentes: alguns fatores determinantes para o trabalho precoce. Rev Lat Am Enferm 2001; 9(3):83-89.

4. Organização Internacional do Trabalho (OIT). Emprego Juvenil: Síntese de Indicadores Sociais 2016. Brasília: Nações Unidas, IBGE; 2021.

5. Rohlman DS, Nuwayhid I, Ismail A, Saddik B. Using epidemiology and neurotoxicology to reduce risks to young workers. Neurotoxicology 2012; 33(4):817-822.

6. Esin MN, Bulduk S, Ince H. Workrelated risks and health problems of working children in urban Istanbul, Turkey. J Occup Health 2005; 47(5):431-436.

7. Facchini LA, Fassa AG, Dall'Agnol M, Maia MFS. Trabalho infantil em Pelotas: perfil ocupacional e contribuição à economia. Cien Saude Colet 2003; 8(4):953961.

8. Benvegnú LA, Fassa AG, Facchini LA, Wegman DH, Dall'Agnol MM. Work and behavioural problems in children and adolescents. Int J Epidemiol 2005; 34(6):1417-1424.

9. GBD 2015 Obesity Collaborators, Afshin A, Forouzanfar MH, Reitsma MB, Sur P, Estep K, Lee A, Marczak L, Mokdad AH, Moradi-Lakeh M, Naghavi M, Salama JS, Vos T, Abate KH, Abbafati C, Ahmed MB, Al-Aly Z, Alkerwi A, Al-Raddadi R, Amare AT, Amberbir A, Amegah AK, Amini E, Amrock SM, Anjana RM, Ärnlöv J, Asayesh H, Banerjee A, Barac A, Baye E, Bennett DA, Beyene AS, Biadgilign S, Biryukov S, Bjertness E, Boneya DJ, Campos-Nonato I, Carrero JJ, Cecilio P, Cercy K, Ciobanu LG, Cornaby L, Damtew SA, Dandona L, Dandona R, Dharmaratne SD, Duncan BB, Eshrati B, Esteghamati A, Feigin VL, Fernandes JC, Fürst T, Gebrehiwot TT, Gold A, Gona PN, Goto A, Habtewold TD, Hadush KT, Hafezi-Nejad N, Hay SI, Horino M, Islami F, Kamal R, Kasaeian A, Katikireddi SV, Kengne AP, Kesavachandran CN, Khader YS, Khang Y-H, Khubchandani J, Kim D, Kim YJ, Kinfu Y, Kosen S, Ku T, Defo BK, Kumar GA, Larson HJ, Leinsalu M, Liang X, Lim SS, Liu P, Lopez AD, Lozano R, Majeed A, Malekzadeh R, Malta DC, Mazidi M, McAlinden C, McGarvey ST, Mengistu DT, Mensah GA, Mensink GBM, Mezgebe HB, Mirrakhimov EM, Mueller UO, Noubiap JJ, Obermeyer CM, Ogbo FA, Owolabi MO, Patton GC, Pourmalek F, Qorbani M, Rafay A, Rai RK, Ranabhat CL, Reinig N, Safiri S, Salomon JA, Sanabria JR, Santos IS, Sartorius B, Sawhney M, Schmidhuber J, Schutte AE, Schmidt MI, Sepanlou SG, Shamsizadeh M, Sheikhbahaei S, Shin M-J, Shiri R, Shiue I, Roba HS, Silva DAS, Silverberg JI, Singh JA, Stranges S, Swaminathan S, Tabarés-Seisdedos R, Tadese F, Tedla BA, Tegegne BS, Terkawi AS, Thakur JS, Tonelli M, Topor-Madry R, Tyrovolas S, Ukwaja KN, Uthman OA, Vaezghasemi M, Vasankari T, Vlassov VV, Vollset SE, Weiderpass E, Werdecker A, Wesana J, Westerman R, Yano Y, Yonemoto N, Yonga G, Zaidi Z, Zenebe ZM, Zipkin B, Murray CJL. Health Effects of Overweight and Obesity in 195 Countries over 25 Years. N Engl J Med 2017; 377(1):13-27. 
10. Romanzini M, Reichert FF, Lopes AS, Petroski ÉL, Farias Júnior JC. Prevalência de fatores de risco cardiovascular em adolescentes. Cad Saude Publica 2008; 24(11):2573-2581.

11. Hemmingsson T, Lundberg I. Is the association between low job control and coronary heart disease confounded by risk factors measured in childhood and adolescence among Swedish males $40-53$ years of age? Int J Epidemiol 2006; 35(3):616-622.

12. Ribeiro RQC, Lotufo PA, Lamounier JA, Oliveira RG, Soares JF, Botter DA. Fatores adicionais de risco cardiovascular associados ao excesso de peso em crianças e adolescentes. O estudo do coração de Belo Horizonte. Arq Bras Cardiol 2006; 86(6):408-418.

13. Schnall P, Belkić K, Landsbergis P, Baker D. Why the workplace and cardiovascular disease? Occup Med State Art Rev 2000; 15(1):1-6.

14. Marmot M, Theorell T, Siegrist J. Work and coronary heart disease. In: Stansfeld SA, Marmot MG. Stress and the heart: Psychosocial pathways to coronary heart disease. BMJ Books; 2002. p. 50-71.

15. Vasconcellos MTL, Silva PLN, Szklo M, Kuschnir MCC, Klein CH, Abreu GDA, Barufaldi LA, Bloch KV Desenho da amostra do Estudo do Risco Cardiovascular em Adolescentes (ERICA). Cad Saude Publica 2015; 31(5):1-10.

16. Bloch KV, Szklo M, Kuschnir MCC, Abreu GA, Barufaldi LA, Klein CH, Vasconcelos MTL, Veiga GV, Figueiredo VC, Dias A, Moraes AJP, Souza ALL, Oliveira AMA, D'Argord Schaan B, Tavares BM, Oliveira CL, Cunha CF, Giannini DT, Belfort DR, Ribas DLB, Santos EL, Leon EB, Fujimori E, Oliveira ERA, Magliano ES, Vasconcelos FAG, Azevedo GD, Brunken GS, Dias GM, Correa Filho HR, Monteiro MI, Guimarães ICB, Faria Neto JR, Oliveira JS, Carvalho KMB, Gonçalves LGO, Santos MM, Muniz PT, Jardim PCBV, Ferreira PAM, Montenegro Jr RM, Gurgel RQ, Vianna RP, Vasconcelos SM, Matta SS, Martins SMS, Goldberg TBL, Silva TLN. The study of cardiovascular risk in adolescents - ERICA: Rationale, design and sample characteristics of a national survey examining cardiovascular risk factor profile in Brazilian adolescents. BMC Public Health 2015; 15:94.

17. Sallis JF, Strikmiller PK, Harsha DW, Feldman HA, Ehlinger S, Stone EJ, Williston J, Woods S. Validation of interviewer- and self-administered physical activity checklists for fifth grade students. Med Sci Sports Exerc 1996; 28(7):840-851.

18. Farias Júnior JC, Lopes AS, Mota J, Santos MP, Ribeiro JC, Hallal PC. Validade e reprodutibilidade de um questionário para medida de atividade física em adolescentes: uma adaptação do Self-Administered Physical Activity Checklist. Rev Bras Epidemiol 2012; 15(1):198-210.

19. World Health Organisation (WHO). Global recommendations on physical activity for health. Geneva: WHO; 2010

20. Cureau FV, Silva TLN, Bloch KV, Fujimori E, Belfort DR, Carvalho KMB, Leon EB, Vasconcellos MTL, Ekelund U. ERICA: leisure-time physical inactivity in Brazilian adolescents. Rev Saude Publica 2016; 50(Supl. 1):4s.
21. Figueiredo VC, Szklo AS, Costa LC, Kuschnir MCC Silva TLN, Bloch KV, Szklo M. ERICA: prevalência de tabagismo em adolescentes brasileiros. Rev Saude Publica 2016; 50(Supl. 1):12s.

22. Warren CW, Jones NR, Peruga A, Chauvin J, Baptiste JP, Silva VC, el Awa F, Tsouros A, Rahman K, Fishburn B, Bettcher DW, Asma S, Centers for Disease Control and Prevention (CDC). Global youth tobacco surveillance, 2000-2007. MMWR Surveill Summ 2008; 57(1):1-28.

23. Coutinho ESF, França-Santos D, Magliano ES, Bloch KV, Barufaldi LA, Cunha CF, Vasconcellos MTL, Szklo M. ERICA: padrões de consumo de bebidas alcoólicas em adolescentes brasileiros. Rev Saude Publica 2016; 50(Supl. 1):8s.

24. Abreu GA, Silva TLN, Teixeira LR, Bloch KV. Análise da qualidade da informação autorreferida sobre duração do sono de escolares do Estudo de Riscos Cardiovasculares em Adolescentes (ERICA). Cad Saude Publica 2019; 35(10):e00152918.

25. Bloch KV, Klein CH, Szklo M, Kuschnir MCC, Abreu GA, Barufaldi LA, Veiga GV, Schaan B, Silva TLN, Vasconcellos MTL, Moraes AJP, Borges AL, Oliveira AMA, Tavares BM, Oliveira CL, Cunha CF, Giannini DT, Belfort DR, Santos EL, Leon EB, Fujimori E, Oliveira ERA, Magliano ES, Vasconcelos FAG, Azevedo GD, Brunken GS, Guimarães ICB, Faria Neto JR, Oliveira JS, Carvalho KMB, Gonçalves LGO, Monteiro MI, Santos MM, Jardim PCBV, Ferreira PAM, Montenegro Jr RM, Gurgel RQ, Vianna RP, Vasconcelos SM, Goldberg TBL. ERICA: prevalences of hypertension and obesity in Brazilian adolescents. Rev Saude Publica 2016; 50(Supl. 1):9s.

26. Onis M, Onyango AW, Borghi E, Siyam A, Nishida C, Siekmann J. Development of a WHO growth reference for school-aged children and adolescents. Bull World Heal Organ 2007; 85(9):660-667.

27. National High Blood Pressure Education Program Working Group on High Blood Pressure in Children and Adolescents. The fourth report on the diagnosis, evaluation, and treatment of high blood pressure in children and adolescents. Pediatrics 2004; 114(2):555576.

28. Cureau FV, Bloch KV, Henz A, Schaan CW, Klein CH, Oliveira CL, Giannini DT, Leon EB, Abreu GA, Telo GH, Dias GM, Carvalho KMB, Barufaldi LA, Kuschnir MCC, Szklo M, Montenegro Jr R, Silva TLN, Ekelund U, Schaan BD. Challenges for conducting blood collection and biochemical analysis in a large multicenter school-based study with adolescents: lessons from ERICA in Brazil. Cad Saude Publica 2017; 33(4):e00122816.

29. Faria-Neto JR, Bento VFR, Baena CP, Olandoski M, Gonçalves LGO, Abreu GA, Kuschnir CC, Bloch KV. ERICA: prevalência de dislipidemia em adolescentes brasileiros. Rev Saude Publica 2016; 50(Supl. 1):10s. 
30. Kuschnir MCC, Bloch KV, Szklo M, Klein CH, Barufaldi LA, Abreu GA, Schaan B, Veiga GV, Silva TLN, Vasconcellos MTL, Moraes AJP, Borges AL, Oliveira AMA, Tavares BM, Oliveira CL, Cunha CF, Giannini DT, Belfort DR, Santos EL, Leon EB, Fujimori E, Oliveira ERA, Magliano ES, Vasconcelos FAG, Azevedo GD, Brunken GS, Guimarães ICB, Faria Neto JR, Oliveira JS, Carvalho KMB, Gonçalves LGO, Monteiro MI, Santos MM, Muniz PT, Jardim PCBV, Ferreira PAM, Montenegro Jr RM, Gurgel RQ, Vianna RP, Vasconcelos SM, Martins SMS, Goldberg TBL. ERICA: prevalence of metabolic syndrome in Brazilian adolescents. Rev Saude Publica 2016; 50(Supl. 1):11s.

31. Santana VS, Itaparica MS. Social contextual factors contributing to child and adolescent labor: an ecological analysis. Rev Saude Publica 2011; 45(4):676-684.

32. Gonçalves H, Menezes AMB, Bacchieri G, Dilélio AS, Bocanegra CAD, Castilhos ED, Gallo EAG, Fiori EJFNS, Meucci RD, Araújo CLP, Carvalho S. Perfil de trabalho urbano de adolescentes de 14-15 anos: um estudo populacional no Sul do Brasil. Cien Saude Colet 2012; 17(5):1267-1274.

33. Souza DPO, Areco KN, Silveira Filho DX. Álcool e alcoolismo entre adolescentes da rede estadual de ensino de Cuiabá, Mato Grosso TT. Rev Saude Publica 2005; 39(4):585-592.

34. Standing G. The international labour organization. New Polit Econ 2010; 15(2):307-318.

35. Boo FL, Mateus MC, Duryea S. Analysis of socioeconomic gradients in the development of children aged 0-3 years in Fortaleza, Northeastern Brazil. Rev Saude Publica 2018; 52:84.

36. Teixeira LR, Fischer FM, Nagai R, Turte SL. Teen at Work: The Burden of a Double Shift on Daily Activities. Chronobiol Int 2004; 21(6):845-858.

37. Carvalho IMM. Algumas lições do Programa de Erradicação do Trabalho Infantil. São Paulo Perspect 2004; 18(4):50-61.

38. Bernardes JA. Novas fronteiras do capital no Cerrado: dinâmica e contradições da expansão do agronegócio na Região Centro-Oeste, Brasil. Scripta Nova 2015; 21(507):741-798.

39. Drake KM, Beach ML, Longacre MR, MacKenzie T, Titus LJ, Rundle AG, Dalton MA. Influence of Sports, Physical Education, and Active Commuting to School on Adolescent Weight Status. Pediatrics 2012; 130(2):e296-304.

40. Wickel EE, Eisenmann JC. Contribution of youth sport to total daily physical activity among 6- to 12-yr -old boys. Med Sci Sports Exerc 2007; 39(9):1493-1500.

41. Sirimarco PL. Fatores associados ao uso pesado de álcool entre estudantes das capitais brasileiras. Rev Saude Publica 2010; 44(2):267-273.

42. Romano M. Pesquisa de compra de bebidas alcoólicas por adolescentes em duas cidades do estado de São Paulo. Rev Saude Publica 2007; 41(4):495-501.

43. Felden EPG, Barbosa DG, Ferrari GJ, Santos MO, Pelegrini A, Silva DAS. Factors associated with sleep duration in Brazilian high school students. Chronobiol Int 2017; 34(6):773-781.
44. Fischer FM, Wey D, Valente D, Luz AA, Pinheiro F, Fonseca BC, Silva-Costa A, Moreno CR, Menna-Barreto L, Teixeira LR. Sleep patterns and sleepiness among young students: a longitudinal study before and after admission as trainees and apprentices. Chronobiol Int 2015; 32(4):478-485.

45. Ferrie JE, Shipley MJ, Cappuccio FP, Brunner E, Miller MA, Kumari M, Marmot MG. A prospective study of change in sleep duration: Associations with mortality in the Whitehall II cohort. Sleep 2007; 30(12):16591666.

46. Carskadon MA, Acebo C, Jenni OG. Regulation of adolescent sleep: Implications for behavior. Ann N Y Acad Sci 2004; 1021:276-291.

47. Kenney SR, LaBrie JW, Hummer JF, Pham AT. Global sleep quality as a moderator of alcohol consumption and consequences in college students. Addict Behav 2012; 37(4):507-512.

48. Telzer EH, Fuligni AJ, Lieberman MD, Galván A. The effects of poor quality sleep on brain function and risk taking in adolescence. Neuroimage 2013; 71:275-283.

49. McCrindle BW. Assessment and management of hypertension in children and adolescents. Nat Rev Cardiol 2010; 7(3):155-163.

50. Cao ZQ, Zhu L, Zhang T, Wu L, Wang Y. Blood pressure and obesity among adolescents: A school-based population study in China. Am J Hypertens 2012; 25(5):576-582.

51. Camhi SM, Katzmarzyk PT. Tracking of cardiometabolic risk factor clustering from childhood to adulthood. Int J Pediatr Obes 2010; 5(2):122-129.

52. Penna G. Pesquisa Nacional de Saúde do Escolar (PeNSE). Cien Saude Colet 2010; 15(Supl. 2):3006.

Artigo apresentado em 17/02/2020

Aprovado em 19/04/2021

Versão final apresentada em 21/04/2021

Editores-chefes: Romeu Gomes, Antônio Augusto Moura da Silva 